\title{
Utility of qualitative C- reactive protein assay and white blood cells counts in the diagnosis of neonatal septicaemia at Bugando Medical Centre, Tanzania
}

Flora Chacha ${ }^{1 \dagger}$, Mariam M Mirambo ${ }^{2 \dagger}$, Martha F Mushi ${ }^{2}$ Neema Kayange ${ }^{1}$, Antke Zuechner ${ }^{1}$, Benson R Kidenya ${ }^{3}$ and Stephen E Mshana ${ }^{2^{*}}$

\begin{abstract}
Background: Neonatal septicaemia diagnosis based on clinical features alone is non-specific leading to the initiation of unnecessary antibiotic treatment posing a danger of increased antibiotic resistance. In the present study the utility of serial qualitative C-reactive protein (CRP) assay and white blood cells count (WBC) in the diagnosis of neonatal septicaemia was investigated using blood culture as gold standard.

Methods: A total of 305 neonates admitted at Bugando Medical Centre (BMC) neonatal units between September 2013 and April 2014 were enrolled. Demographic and clinical data were collected using standardized data collection tool. Blood specimens were collected for blood culture, WBC count and qualitative CRP assay.

Results: Of 305 neonates; 224 (73.4\%) were $\leq 72$ hrs of age and 91 (29.8\%) had low birth weight. The positive CRP assay was observed in 67 (22.0\%), 80 (26.2\%) and 88 (28.9\%) of neonates on day 1, 2 and 3 respectively; with any CRP positive occurred in 104 (34.1\%) of neonates. The sensitivities of CRP assay in the diagnosis of septicaemia using culture as gold standard on day 1, 2, 3 and any positive were $40.4 \%, 53.2 \%, 54.8 \%$ and $62.9 \%$ respectively. While specificities were $82.7 \%, 80.7 \%, 77.8 \%$ and $73.3 \%$ respectively. Higher sensitivity of $75 \%$ was observed when CRP was used to diagnose gram negative septicaemia compared to $50 \%$ that was observed in the diagnosis of gram positive septicaemia. WBC count of $\geq 13 \times 10^{9} / \mathrm{L}$ had sensitivity and specificity of $64.5 \%$ and $66.7 \%$ respectively with area under the curve of 0.694 . When the any positive CRP and WBC of $\geq 13 \times 10^{9} / \mathrm{L}$ were used the sensitivity increased to $90.3 \%$ with specificity of $50 \%$. Neonates with septicaemia due to gram negative bacteria were significantly found to have higher rates of positive CRP than neonates with gram positive septicaemia and with negative culture ( $p<0.001$, OR 8.2, 95 Cl; 2.9-26).
\end{abstract}

Conclusion: In place where blood culture is limited neonates having clinical features of neonatal sepsis with positive qualitative CRP assay and increased WBC should urgently be initiated on appropriate sepsis management in order to reduce morbidity and mortality associated with neonatal sepsis.

Keywords: C-reactive protein, Neonatal sepsis, WBC

\footnotetext{
* Correspondence: mshana72@yahoo.com

${ }^{+}$Equal contributors

${ }^{2}$ Department of Microbiology/Immunology Weill Bugando School of

Medicine, Catholic University of Health and Allied Sciences (CUHAS), P.O.

BOX 1464, Mwanza, Tanzania

Full list of author information is available at the end of the article
} 


\section{Background}

Neonatal deaths account for about $40 \%$ of all deaths among underfives. Out of all neonatal deaths in developing countries, $50 \%$ occur during the first 24 hours of life and $75 \%$ during the first week of life [1]. Globally, deaths occurring in the first month of life have increased from $36 \%$ in 1990 to $43 \%$ in 2011. Most of deaths have been due to neonatal septicaemia and prematurity [2]. In 2010 at the Bugando medical center (BMC) prevalence of neonatal septicaemia was 39\% with mortality rate of $19 \%$ [3]. Delayed diagnosis and inappropriate treatment of neonatal septicaemia has been associated with neurological complication with increased mortality [4].

Though blood culture is the gold standard in the diagnosis of neonatal septicaemia, it takes more than 3 days for the final results to be available and the technique is not available in many settings in developing countries [5]. This necessitates the use of antibiotics with no supporting microbiological results; hence leading to unnecessary cost and risk of increased resistance development. In developing countries, there are no suitable clinical or laboratory parameters available to guide the duration of the antibiotic treatment. At BMC and in many other centres in developing countries, full antibiotics courses are prescribed in all neonates suspected with septicaemia regardless of culture results. This practice at $\mathrm{BMC}$ does not reduce the mortality due to neonatal septicaemia however it has been found to add to the problem of antibiotic resistance, as evidenced by the fact that all Klebsiella pneumoniae isolated in the neonatal unit were resistant to gentamicin and being more than $50 \%$ resistant to third generation cephalosporin [6].

Early diagnosis followed by appropriate treatment of all newborns with clinical suspicion of septicaemia has been found to be an important strategy in preventing life threatening complications [7]. Most of the time, initial diagnosis of neonatal septicaemia is based on clinical features which are non-specific; resulting in initiation of unnecessary empirical antibiotic treatment posing to a danger of increased antibiotic resistance [8].

Therefore; in order to guide the empiric antibiotic treatment it is important to evaluate cheap and inexpensive CRP assay as a rapid test to justify the use and duration of antibiotics treatment in many settings in developing countries. C-reactive protein is an acute-phase reactant protein synthesized by the liver within six hours after the onset of infectious process $[9,10]$. There is variation in the performance of CRP in the diagnosis of septicaemia depending on the etiology of septicaemia and the setting. Therefore this study aimed at evaluating the use of serial qualitative CRP assay as a rapid test to accurately predict neonatal septicaemia so as to avoid unnecessary use of antibiotics and to guide the duration of antibiotic therapy.

\section{Methods}

\section{Study design and study area}

This was a hospital based analytical cross sectional study conducted from October 2013 to April 2014. This study was conducted at BMC, Mwanza, Tanzania which is a tertiary teaching hospital serving about 14 million population.

\section{Inclusion criteria and exclusion criteria}

All neonates with clinical suspicion of neonatal sepsis according to WHO criteria [11] admitted at NICU and premature Unit were enrolled. Neonates with history of use of antibiotics before enrolment for more than 72 hours and those with body weight less than 1 kilogram were excluded from the study.

\section{Sample size and sampling procedure}

Sample size was estimated using Buderer formula [12]; using anticipated sensitivity and specificity of $95 \%$ and neonatal sepsis prevalence of $40 \%$ [3]. The minimum sample size obtained was 305 neonates. All neonates admitted to the neonatal wards with clinical sepsis were recruited serially into the study until the sample size was reached. Using WHO guidelines for sepsis in young infants a standard structured data collection tool was designed and used to obtain social demographic data and other relevant factors related to neonatal septicaemia like maternal fever, premature rupture of membrane (PROM), mode of delivery, birth weight of the baby, gestational age (less than 37 completed weeks was considered as premature), temperature of the infant, respiratory rate, cyanosis, jaundice, umbilical redness, convulsion, decreased movement and ability to breast feed.

\section{Laboratory procedures}

\section{$C$ - reactive protein assay}

C-reactive protein was tested qualitatively using Immunopak (RECKON DIAGNOSTICS, INDIA). About $0.3 \mathrm{ml}$ or $0.5 \mathrm{ml}$ of venous blood was collected at 24 hours, 48 hours and 72 hours after admission using plain bottles (BD Vacutainer, Nairobi, Kenya). Assays were done following manufacturer instructions; presence of agglutination similar to positive control was considered as positive CRP assay indicating CRP level of more than $6 \mathrm{mg} / \mathrm{dl}$.

\section{Blood culture}

Blood culture was performed using Brain Heart Infusion broth (BHI) (Oxoid Ltd) in a ratio of blood to BHI of 1:10 as previously described [3]. Subsequent sub-culture was done on day 1, 3 and 7 on 5\% sheep blood agar, chocolate agar and MacConkey agar (Oxoid, UK). Identification of bacteria was performed using conventional physiological and biochemical methods [13,14]. Repeat blood culture was ordered in all cases where Coagulase negative staphylococcus (CNS) was isolated. Re-isolation of CNS was considered significant blood culture result. Antimicrobial susceptibility 
of isolates was determined using disk diffusion method according to the Clinical Laboratory standard Institute (CLSI) [15].

\section{Complete blood count}

About $2 \mathrm{ml}$ of blood in EDTA container (BD Vacutainer, Nairobi, Kenya) was collected for WBC count and platelet count and estimated using hematological analyzer (Beckman coulter (UK) LTD). WBC count of less than $9 \times 10^{9} / 1$ or more than $30 \times 10^{9} / 1$ were considered as leucopenia and leukocytosis respectively [16].

\section{Data analysis}

Data were double entered into Microsoft excel and analyzed using STATA version 11. Results were summarized using proportions (\%) for categorical data and means (SD) or medians (IQR) for continuous variables. Categorical variables were compared using either Pearson's Chisquared or Fisher's exact test where appropriate. The continuous variables were compared using student $t$-test and Wilcoxon sign rank test for parametric and nonparametric variables respectively. To determine the sensitivity and specificity of the CRP in the diagnosing neonatal septicaemia we used 2-by-2 contingency tables. We used Receiver operating characteristic (ROC) to determine the performance $\mathrm{WBC}$ in the diagnosis of neonatal septicemia. While, to determine predictors of positive CRP, univariate followed by multivariate logistic regressions analysis were performed. Predictors investigated included; socio-demographic factors, clinical features and laboratory parameters. Odds ratios with respective 95\% confidence interval $(\mathrm{CI})$ were reported. Predictors with a $p$-value of less than 0.05 were considered statistically significant.

\section{Quality control}

Data from questionnaires were entered into a data sheet. The reading of CRP test was done by two qualified laboratory technologists to avoid bias. All microbiological testing were controlled using quality control strains; Escherichia coli ATCC 25922, Klebsiella pneumoniae ATCC 13883 and Staphylococcus aureus ATCC 25923.

\section{Ethical considerations}

The proposal of this study was presented to the CUHASBugando/BMC department of Pediatric and Child Health for approval and then to CUHAS-Bugando ethics committee for clearance. Written Informed consent for the participation in the study was obtained from mother/caretaker of the respective neonate.

\section{Results}

Baseline characteristics of patient enrolled in the study During the study period a total of 624 neonates were admitted at NICU and premature neonatal unit. Out of 310 neonates with clinical suspicion of sepsis, 305 (98\%) were enrolled in the study from September 2013 to April 2014. Of 305 neonates, 224(73.4\%) were $\leq 72$ hours of age (Table 1). Median age was 1 day with IQR of 1-4 days. Among 305 neonates; 149(48.9\%) were male and 156 (51.2\%) were female. A total of 69 (22.6\%) neonates were premature. Regarding place of delivery, 30(9.8\%) of neonates were delivered at home (Table 1$)$. Thirty one (10.2\%) of the neonates had history of convulsions, 59(19.3\%) had

Table 1 Distribution of demographic characteristics of neonates with sepsis

\begin{tabular}{|c|c|c|}
\hline Child's characteristic & Number & Percent (\%) \\
\hline \multicolumn{3}{|l|}{ Sex } \\
\hline Female & 149 & 48.9 \\
\hline Male & 156 & 51.2 \\
\hline \multicolumn{3}{|l|}{ Age } \\
\hline$\leq 72$ hours & 224 & 73.4 \\
\hline$>72$ hours & 81 & 26.6 \\
\hline \multicolumn{3}{|l|}{ Mode of delivery } \\
\hline Caesarean section & 67 & 22 \\
\hline Spontaneous vertex delivery & 238 & 78 \\
\hline \multicolumn{3}{|l|}{ Birth weight } \\
\hline Very low birth weight & 18 & 5.9 \\
\hline Low birth weight & 73 & 23.9 \\
\hline Normal birth weight & 214 & 70.2 \\
\hline \multicolumn{3}{|l|}{ Gestation age } \\
\hline Premature & 69 & 22.6 \\
\hline Full term & 236 & 77.4 \\
\hline \multicolumn{3}{|l|}{ Hospital delivery } \\
\hline Yes & 275 & 90.2 \\
\hline No & 30 & 9.8 \\
\hline \multicolumn{3}{|l|}{ Convulsion } \\
\hline Yes & 31 & 10.2 \\
\hline No & 274 & 89.8 \\
\hline \multicolumn{3}{|l|}{ Jaundice } \\
\hline Yes & 59 & 19.3 \\
\hline NO & 249 & 80.7 \\
\hline \multicolumn{3}{|l|}{ Poor feeding } \\
\hline Yes & 182 & 59 \\
\hline No & 123 & 40.3 \\
\hline \multicolumn{3}{|l|}{ Oxygen saturation } \\
\hline$<90 \%$ & 133 & 43.6 \\
\hline$>90 \%$ & 172 & 56.4 \\
\hline \multicolumn{3}{|l|}{ Body temperature } \\
\hline Hypothermia & 96 & 31.5 \\
\hline Normal & 23 & 7.5 \\
\hline Hyperthermia & 186 & 61.0 \\
\hline
\end{tabular}


jaundice, $186(61 \%)$ had body temperature of more than $37.5^{\circ} \mathrm{C}$ and $133(43.6 \%)$ had oxygen saturation of less than $90 \%$.

\section{C-reactive protein and blood culture results}

Out of 305 neonates; 104(34.1\%) had any positive CRP; the positive CRP on day 1,2 and 3 were $67(22 \%) 80$ (26.2\%) and $88(28.9 \%)$ respectively. Positive aerobic blood culture was detected in 62(20.3\%) of neonates (Table 2). All specimens with positive culture were detected within $48 \mathrm{hrs}$ of incubation.

\section{Sensitivity, specificity, PPV and NPV of qualitative CRP assay and WBC}

The sensitivity of CRP was found to be $40.4 \%, 53.2 \%$ and $54.8 \%$ on day 1,2 and 3 with specificity of $82.7 \%, 80.7 \%$ and $77.8 \%$ respectively. While the positive predictive value was found to be $37.5 \%, 41.3 \%$ and $38.6 \%$ with negative predictive value of $84.5 \%, 87.1 \%$, and $88.6 \%$ on day 1,2 and 3 respectively. Any positive CRP had sensitivity of $62.9 \%$ with specificity of $73.3 \%$ (Table 2 ). Higher sensitivity was obtained when CRP was used to diagnose gram negative septicaemia than in the diagnosis of gram positive septicaemia ( $75 \%$ vs. $50 \%$ ) with the same specificity. Using WBC cut off point of $\geq 13 \times 10^{9} / \mathrm{L}$ the sensitivity obtained was of $64.5 \%$ with specificity of $66.7 \%$ and area under the curve of 0.6924 (Figure 1). When any positive CRP was combined with raised WBC of $\geq 13 \times 10^{9} / \mathrm{L}$ the sensitivity and specificity obtained were $90.3 \%$ and $50.2 \%$ respectively (Table 2).

\section{C-reactive protein, WBC and neonatal sepsis}

Higher rates of CRP positive were observed among neonates with confirmed neonatal sepsis than those with negative culture $(\mathrm{p}<0.05)$ Figure 2 . Neonates with gram negative sepsis had significantly higher rates of positive CRP than neonates with gram positive neonatal sepsis and neonates with negative blood culture. Significantly higher means of WBC were observed among neonates with positive blood culture and those with positive CRP when compared to neonates with negative culture and negative CRP. Means WBC among neonates with gram negative septicaemia and gram positive septicaemia were $20431.25 \mathrm{c} / \mathrm{mm}^{3}$ and $20525.26 \mathrm{c} / \mathrm{mm}^{3}$ respectively compared to the mean of $13915.72 / \mathrm{mm}^{3}$ among children with negative blood culture (Table 3 ).

\section{Predictors of positive CRP and neonatal septicaemia}

On univariate analysis; the predictors of positive CRP were found to be older age $(\mathrm{p}=0.03)$ and higher body temperature $(\mathrm{p}=0.02)$, however on multivariate analysis only poor feeding and raised body temperature remained significantly associated with positive CRP (Table 4). Factors found to predict neonatal septicaemia were positive CRP ( $<<0.001,95 \%$ CI; 2.6-8.2), elevated WBC $(\mathrm{p}<$ $0.001,95 \% \mathrm{CI} ; 1.03-1.08)$, high body temperature ( $\mathrm{p}=$ 0.04, CI 1.01-1.32) and home delivery 95\% CI; 2.29(1.055.01). Of 305 neonates, 22(7.2\%) died during the study period. Meconium aspiration (OR 3.3, 95\% CI (1.1-10.5), $\mathrm{p}=0.038$ ), convulsions (OR 5.4, 95\% CI (1.8-16.2), $\mathrm{p}=$ 0.003), oxygen desaturation $<90 \%$ (OR 4.7 (CI 1.3-16.8) $\mathrm{p}=0.017)$ and jaundice (OR 7.8(CI 2.3-26.9) $\mathrm{p}=0.001$ were independent factors found to be associated with death. Neonates with positive CRP had 1.7 times risk of death than those with negative CRP.

\section{Discussion}

\section{Baseline characteristics}

The study involved 305 neonates with suspected neonatal septicaemia attending BMC neonatal units. As in previous studies $[3,17,18]$, most of these neonates were below 72 hrs of age and with low birth weight. The low birth weight in the current study is partially contributed to premature delivery because $22.6 \%$ of neonates were delivered prematurely of whom $29.5 \%$ had low birth weight. In contrast to previous study which observed home delivery in $38 \%$ of neonates with suspected neonatal sepsis in the current study only $9.8 \%$ of neonates were delivered at home. From 2010 to 2012, we observed a decrease in home delivery of more than $70 \%$; this could be due to ongoing campaigns by NGOs and

Table 2 Sensitivity, Specificity, Positive and negative predictive values

\begin{tabular}{lllll}
\hline Parameters & Sensitivity & Specificity & PPV & NPV \\
\hline Any CRP + ve & $62.9 \%(49.7-74.8)$ & $73.3 \%(67.2-78.7)$ & $37.5 \%(28.2-47.5)$ & $88.6 \%(83.3-92.6)$ \\
CRP day 1 & $40.3 \%(28.1-53.6)$ & $82.7 \%(77.4-87.3)$ & $37.5 \%(25.8-50.0)$ & $84.5 \%(79.2-88.8)$ \\
CRP day 2 & $53.2 \%(40.1-66.0)$ & $80.7 \%(75.1-85.4)$ & $41.3 \%(30.4-52.8)$ & $87.1 \%(82.0-91.2)$ \\
CRP day 3 & $54.8 \%(41.7-67.5)$ & $77.8 \%(72.0-82.8)$ & $38.6 \%(28.4-49.6)$ & $87.1 \%(81.9-91.3)$ \\
WBC & $64.5 \%(51.3-76.3)$ & $66.7 \%(60.4-72.6)$ & $33.1(24.8-42.2)$ & $88.4(82.5-92.4)$ \\
Any CRP + WBC & $90.3 \%(80.1-96.4)$ & $50.2 \%(43.7-56.7)$ & $31.6 \%(24.9-39.0)$ & $95.3 \%(90.1-98.3)$ \\
CRP gram negative sepsis & $75.0 \%(53.3-90.2)$ & $73.3 \%(67.8-78.7)$ & $21.7 \%(13.4-32.1)$ & $96.7 \%(93.0-98.8)$ \\
CRP gram positive sepsis & $55.3 \%(38.3-71.4)$ & $73.2 \%(67.2-78.7)$ & $24.4 \%(15.8-34.9)$ & $91.3 \%(86.4-94.8)$ \\
\hline
\end{tabular}




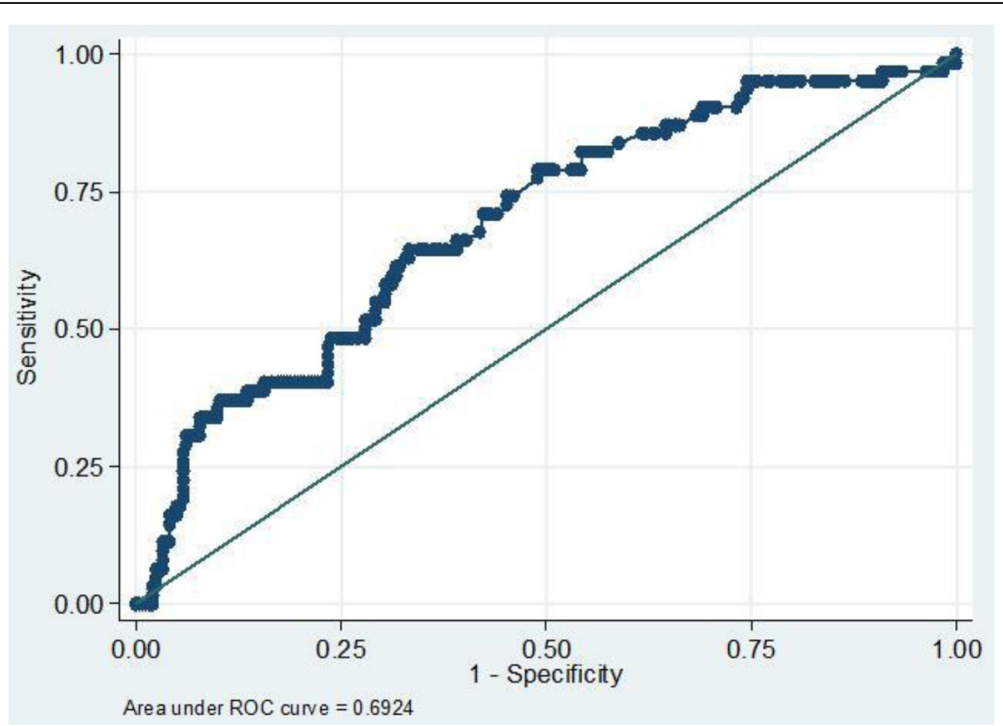

Figure 1 Receiver operating characteristic (ROC) of WBC and blood culture showing the performance WBC in the diagnosis of neonatal septicemia. Cut off points of WBC $\geq 13^{*} 10^{9} / \mathrm{L}$ has sensitivity of $64.5 \%$ and specificity $66.7 \%$.

Ministry of Health Tanzania resulting in awareness of health services and importance of hospital delivery.

\section{Clinical presentation of neonates}

Clinical features of neonatal sepsis are usually non-specific and subtle and neonates with suspected sepsis can present with one or more of the following; fever, jaundice, convulsion, lethargy, poor feeding etc. Almost similar rates clinical findings (poor feeding, cyanosis, jaundice, body temperature, lethargy, chest in drawing) were observed in this study as in other studies in Tanzania $[3,18]$. This could be explained by the fact that similar inclusion criteria were used to enroll study participants. Compare to previous study in the same setting 4 years ago, low convulsion rate was observed in the present study, this could be

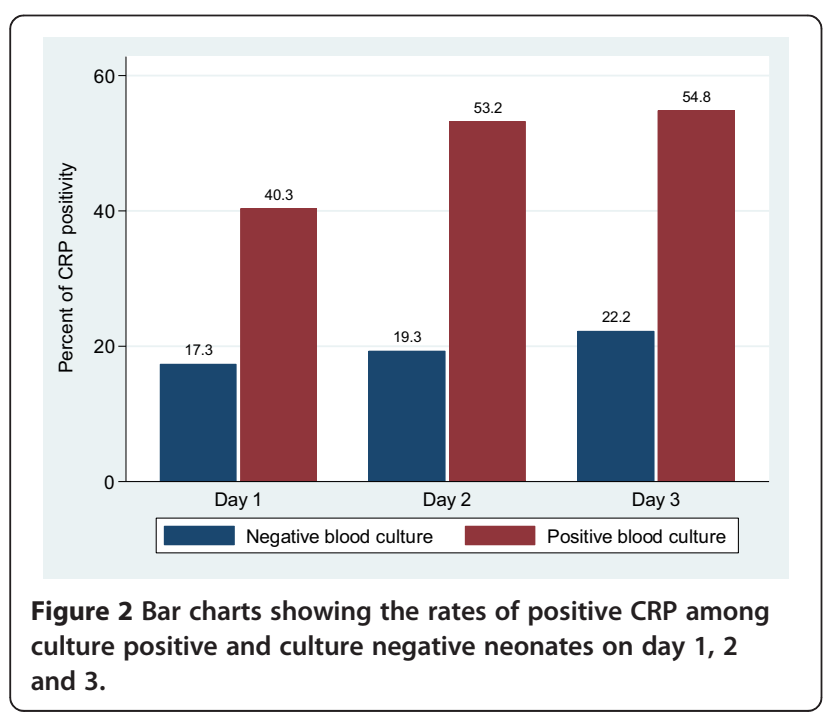

due to less birth asphyxia in present study since most neonates were hospital delivery and also there are improvements in NICU care especially in checking random blood glucose to prevent hypoglycemia.

\section{Utility of qualitative CRP assay and WBC count in the diagnosis of neonatal septicaemia}

In the present study, the overall sensitivity and specificity of CRP in the diagnosis of neonatal septicaemia were $63 \%$ and $73 \%$ respectively. Almost similar findings were observed in Nigeria whereby sensitivity and specificity of $74 \%$ and $74.1 \%$ respectively were observed using semiquantitative assay [19]. The slightly difference could be explained by the type of the tests used, in Nigeria study they used Lorne CRP latex kit from Great Britain while in the current study the semi-quantitative assay from Reckon Diagnostic, India, Immunopak was used. Low sensitivity and specificity are observed when our results are compared with other studies $[10,20,21]$ which used quantitative assays.

There are no established references intervals of CRP in the neonatal period and upper limit have been established only in symptomatic neonates. CRP may rise physiologically after stressful delivery, intraventricular hemorrhage, fetal distress, perinatal asphyxia and meconium aspiration. In these conditions the CRP level usually goes back to normal within 24-48 hours. These conditions contribute to the reduced specificity of the qualitative assay of CRP in the diagnosis of neonatal septicaemia. In these situations, a single normal value is not sufficient to diagnose neonatal septicaemia, therefore to increase specificity serial assays are recommended. In most of the time neonates with persistent high CRP levels are more likely to have 
Table 3 CRP, Neonatal sepsis and WBC in relation to gram reactions

\begin{tabular}{|c|c|c|c|c|}
\hline CRP & $\%$ CRP positive & OR & $95 \% \mathrm{Cl}$ & $P$ value \\
\hline \multicolumn{5}{|l|}{ CRP1 } \\
\hline Negative culture (243) & $42(17.3 \%)$ & 1 & & \\
\hline Gram positive sepsis (38) & $12(31.5 \%)$ & 2.2 & $0.93-4.9$ & 0.0375 \\
\hline Gram negative sepsis (24) & $13(54.4 \%)$ & 5.6 & $2.1-14.87$ & $<0.0001$ \\
\hline \multicolumn{5}{|l|}{ CRP2 } \\
\hline Negative culture (243) & 47 (19.3\%) & 1 & & \\
\hline Gram positive sepsis (38) & $17(44.7 \%)$ & 3.4 & $1.5-7.2$ & 0.0005 \\
\hline Gram negative sepsis (24) & $16(66.7 \%)$ & 8.3 & $3.1-23.67$ & $<0.0001$ \\
\hline \multicolumn{5}{|l|}{ CRP3 } \\
\hline Negative culture (243) & $54(22.2 \%)$ & 1 & & \\
\hline Gram positive sepsis (38) & 17 (44.7\%) & 2.8 & $1.2-6.0$ & 0.003 \\
\hline Gram negative sepsis (24) & 17 (70.0\%) & 8.5 & $3.1-25.3$ & $<0.0001$ \\
\hline \multicolumn{5}{|l|}{ Any positive } \\
\hline Negative culture (243) & $65(26.7 \%)$ & 1 & & \\
\hline Gram positive sepsis (38) & $21(55.2 \%)$ & 3.3 & $1.5-7.2$ & 0.0004 \\
\hline Gram negative sepsis (24) & $18(75.0 \%)$ & 8.2 & $2.9-26.2$ & $<0.0001$ \\
\hline Parameter & $\mathrm{N}$ & Mean WBC $\mathrm{c} / \mathrm{mm}^{3}$ & $P$ value & \\
\hline Negative culture & 243 & $13915.72 \pm 11707.4$ & & \\
\hline Gram positive sepsis & 38 & $20525.26 \pm 11800.8$ & 0.0007 & \\
\hline Gram negative sepsis & 24 & $20431.25 \pm 14107.8$ & 0.0056 & \\
\hline \multicolumn{5}{|l|}{ CRP positive } \\
\hline \multicolumn{5}{|l|}{ CRP1 } \\
\hline Positive & 67 & $18025.1 \pm 15964.6$ & & \\
\hline Negative & 238 & $14471.2 \pm 10782.2$ & 0.0173 & \\
\hline \multicolumn{5}{|l|}{ CRP2 } \\
\hline Positive & 80 & $16785.6 \pm 13529.9$ & & \\
\hline Negative & 225 & $14706.6 \pm 11633.8$ & 0.0950 & \\
\hline \multicolumn{5}{|l|}{ CRP3 } \\
\hline Positive & 88 & $16925.8 \pm 12697.2$ & & \\
\hline Negative & 217 & $14573.1 \pm 11914.3$ & 0.0632 & \\
\hline \multicolumn{5}{|l|}{ Any CRP positive } \\
\hline Positive & 104 & $17301.7 \pm 14137.8$ & & \\
\hline Negative & 201 & $14191.3 \pm 10865.0$ & 0.0086 & \\
\hline
\end{tabular}

neonatal sepsis. In the present study there was an increase in positive rates of CRP among neonates from day 1 to day 3 with majority of neonates who were positive on day 1 remained positive on day 3 . These findings suggest that most of our neonates with positive CRP had septicaemia [22]. The discrepancy between CRP and culture results in this study could be explained by the culture technique used. In the present study manual blood culture was used; this technique has been found to contribute to low sensitivity of CRP when compared to automated techniques [23]. However, there has been wide range of CRP sensitivity reported ranging from $47-100 \%$ and this is due to different reference values and test methodologies [23]. Using quantitative methods; CRP assays have been shown to have higher sensitivity than qualitative methods. Other factors that could explain low sensitivity in our study is the predominant of gram positive bacteria; neonates with gram positive septicaemia were found to have significantly lower rates of positive CRP than among neonates with gram negative sepsis. Several studies have reported Creactive protein to be higher in gram negative bacteria than gram positive bacteria neonatal septicaemia [24,25]. In this study when sub-analysis was done the sensitivity of CRP to diagnose gram negative neonatal septicaemia 
Table 4 Factors associated with positive CRP, neonatal sepsis and death on multivariate logistic regression

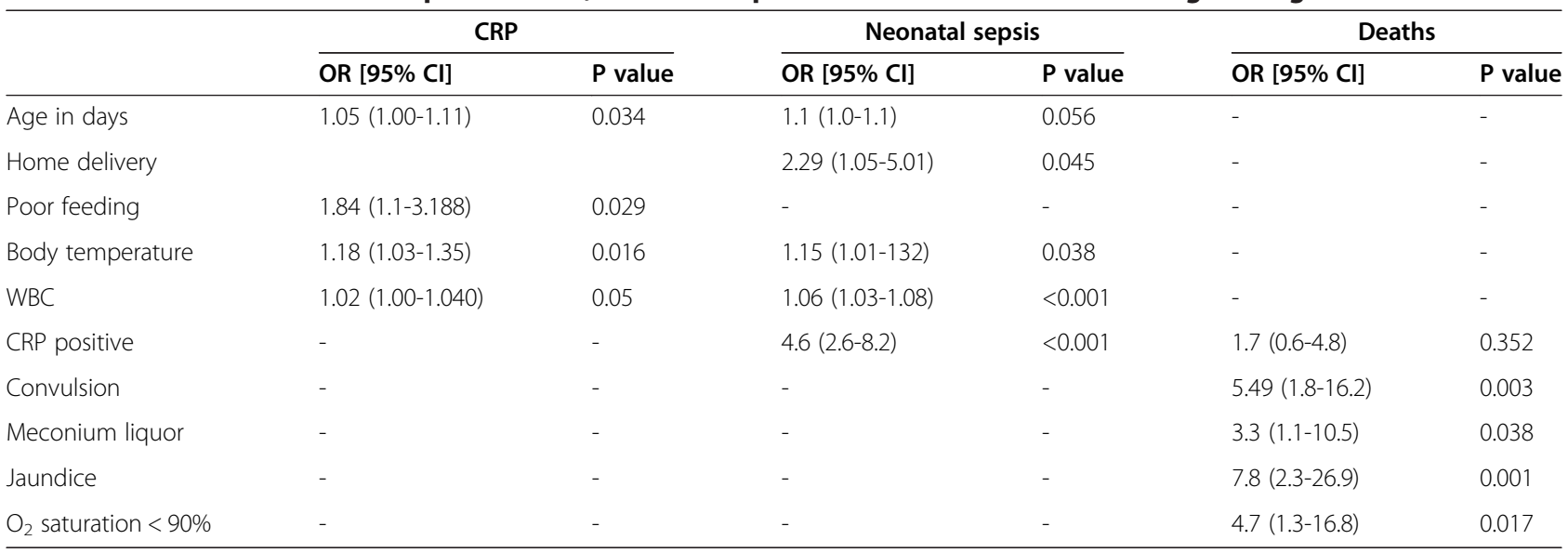

increased to $75 \%$ while for gram positive septicaemia dropped to $50 \%$.

In several previous studies [26-28]; WBC shows little correlation with neonatal septicaemia however in the present study neonates with septicaemia had significantly higher mean of WBC than those with negative culture. The sensitivity of increased WBC of $\geq 13 \times 10^{9} / \mathrm{L}$ in the diagnosis of neonatal septicaemia was comparable to previous studies. When the increased WBC was combined with any positive CRP sensitivity of $90.3 \%$ was obtained similar to a recent study among preterm babies [29].

Including neonates admitted at Bugando Medical Center with clinical suspicion of neonatal sepsis was major limitation of this study. Other limitations include lack of lumbar puncture due to lack of appropriate instruments and/or supplies. Additionally the blood culture was done using only the aerobic manual system again because of lack of equipment and supplies. Finally, there was no control group due to ethical constraints and difficulty obtaining blood in infants who have no clinical suspicious for septicaemia.

\section{Conclusion}

Serial CRP qualitative assays combined with raised WBC has high sensitivity in the diagnosis of neonatal septicaemia. In place where blood culture is limited, neonates with positive qualitative CRP assay and raised WBC together with clinical features such as convulsion, raised body temperature and poor feeding should urgently be initiated on appropriate sepsis management in order to reduce associated morbidity and mortality. Based on these findings CRP and WBC counts can be used as inexpensive methods to diagnose neonatal septicaemia in developing countries in order reduce the duration of antibiotics treatment hence preventing resistance development. Another study using quantitative CRP assay should be considered in our setting in order to estimate the cutoff point of CRP which strongly predicts neonatal septicaemia.
Competing interest

The authors declare that they have no competing interests.

\section{Authors' contributions}

FC, NK, MMM and SEM designed the study. FC, NK, AZ enrolled the patients and collected the samples. FC, BRK, MFM and SEM analyzed the data. FC, MMM and SEM wrote the manuscript which was revised and approved by all co authors.

\section{Acknowledgements}

We are very gratefully for the parents who allow their neonates to participate in the study. We would like to acknowledge the assistance and guidance provided by all staff members of the Department of Pediatrics, Bugando Medical Center, as well as the Department of Microbiology/ Immunology, Catholic University of Health and Allied Sciences, Bugando Hospital. This research was supported by grants from Ministry of Health to FC, reagents from CUHAS to SEM.

\section{Author details}

${ }^{1}$ Department of Pediatric and child Health Weill Bugando School of Medicine, Mwanza, Tanzania. ${ }^{2}$ Department of Microbiology/Immunology Weill Bugando School of Medicine, Catholic University of Health and Allied Sciences (CUHAS), P.O. BOX 1464, Mwanza, Tanzania. ${ }^{3}$ Department of Biochemistry and Molecular Biology, Weill Bugando School of Medicine, Mwanza, Tanzania.

Received: 4 August 2014 Accepted: 29 September 2014 Published: 3 October 2014

\section{References}

1. Vergnano S, Sharland M, Kazembe P, Mwansambo C, Heath P: Neonatal sepsis: an international perspective. Arch Dis Child Fetal Neonatal Ed 2005, 90(3):F220-FF224.

2. Blencowe $\mathrm{H}$, Cousens $\mathrm{S}$ : Review: addressing the challenge of neonatal mortality. Trop Med Int Health 2013, 18(3):303-312.

3. Kayange N, Kamugisha E, Mwizamholya DL, Jeremiah S, Mshana SE: Predictors of positive blood culture and deaths among neonates with suspected neonatal sepsis in a tertiary hospital, Mwanza-Tanzania. BMC Pediatr 2010, 10(1):39.

4. Lim DV, Morales W, Walsh A, Kazanis D: Reduction of morbidity and mortality rates for neonatal group B streptococcal disease through early diagnosis and chemoprophylaxis. J Clin Microbiol 1986, 23(3):489-492.

5. Paes BA, Modi A: Value of superficial cultures: diagnosing neonatal sepsis in a community hospital. Can Fam Physician 1992, 38:1796-1800.

6. Mshana SE, Hain T, Domann E, Lyamuya EF, Chakraborty T, Imirzalioglu C: Predominance of klebsiella pneumoniae ST14 carrying CTX-M-15 causing neonatal sepsis in Tanzania. BMC Infect Dis 2013, 13:466.

7. Volante E, Moretti S, Pisani F, Bevilacqua G: Early diagnosis of bacterial infection in the neonate. J Matern Fetal Neonatal Med 2004, 16(2):13-16. 
8. Thaver D, Zaidi AK: Burden of neonatal infections in developing countries: a review of evidence from community-based studies. Pediatr Infect Dis J 2009, 28(1):S3-S9.

9. Chiesa C, Panero A, Osborn JF, Simonetti AF, Pacifico L: Diagnosis of neonatal sepsis: a clinical and laboratory challenge. Clin Chem 2004, 50(2):279-287.

10. Hofer N, Zacharias E, Mueller W, Resch B: An update on the use of Creactive protein in early-onset neonatal sepsis: current insights and new tasks. Neonatology 2012, 102(1):25-36.

11. Group WYIS: Clinical prediction of serious bacterial infections in young infants in developing countries. Pediatr Infect Dis J 1999, 18(10):S23-S31.

12. Buderer NMF: Statistical methodology: I. Incorporating the prevalence of disease into the sample size calculation for sensitivity and specificity. Acad Emerg Med 1996, 3(9):895-900.

13. Christopher A, Mshana SE, Kidenya BR, Hokororo A, Morona D: Bacteremia and resistant gram-negative pathogens among under-fives in Tanzania. Ital J Pediatr 2013, 39(1):27.

14. Mshana S, Kamugisha E, Miramb M, Chalya P, Rambau P, Mahalu W, Lyamuya E: Prevalence of clindamycin inducible resistance among methicillin-resistant staphylococcus aureus at Bugando Medical Centre, Mwanza, Tanzania. Tanzan J Health Res 2009, 11(2):59-64.

15. Wikler MA, Cockerill FR, Clinical and Laboratory Standards Institute: Performance Standards for Antimicrobial Susceptibility Testing: Eighteenth Informational Supplement. Wayne: Clinical and Laboratory Standards Institute; 2008.

16. Bhandari V, Wang C, Rinder C, Rinder H: Hematologic profile of sepsis in neonates: neutrophil CD64 as a diagnostic marker. Pediatrics 2008, 121(1):129-134.

17. Mugalu J, Nakakeeto MK, Kiguli S, Kaddu-Mulindwa DH: Aetiology, risk factors and immediate outcome of bacteriologically confirmed neonatal septicaemia in Mulago Hospital, Uganda. Afr Health Sci 2006, 6(2):120-126.

18. Mhada TV, Fredrick F, Matee Ml, Massawe A: Neonatal sepsis at Muhimbili national hospital, Dar es salaam, Tanzania; aetiology, antimicrobial sensitivity pattern and clinical outcome. BMC Public Health 2012, 12(1):904.

19. West BA, Peterside O, Ugwu RO, Eneh AU: Prospective evaluation of the usefulness of C-reactive protein in the diagnosis of neonatal sepsis in a sub-Saharan African region. Antimicrob Resist Infect Control 2012, 1(1):22.

20. Nuntnarumit P, Pinkaew O, Kitiwanwanich S: Predictive values of serial C-reactive protein in neonatal sepsis. J Med Assoc Thai 2002, 85(Suppl 4):S1151-1158.

21. Khassawneh M, Hayajneh WA, Kofahi H, Khader Y, Amarin Z, Daoud A: Diagnostic markers for neonatal sepsis: comparing C-reactive protein interleukin-6 and immunoglobulin M. Scand J Immunol 2007, 65(2):171-175.

22. Polin RA: Management of neonates with suspected or proven early-onset bacterial sepsis. Pediatrics 2012, 129(5):1006-1015.

23. Malik A, Hui CP, Pennie RA, Kirpalani H: Beyond the complete blood cell count and C-reactive protein: a systematic review of modern diagnostic tests for neonatal sepsis. Arch Pediatr Adolesc Med 2003, 157(6):511-516.

24. Alexandraki I, Palacio C: Gram-negative versus gram-positive bacteremia: what is more alarming. Crit Care 2010, 14:161.

25. Vallance $\mathrm{H}$, Lockitch $\mathrm{G}$ : Rapid, semi-quantitative assay of C-reactive protein evaluated. Clin Chem 1991, 37(11):1981-1982.

26. Franz AR, Kron M, Pohlandt F, Steinbach G: Comparison of procalcitonin with interleukin 8, C-reactive protein and differential white blood cell count for the early diagnosis of bacterial infections in newborn infants. Pediatr Infect Dis J 1999, 18(8):666-671.

27. Ohlsson A, Vearncombe M: Congenital and nosocomial sepsis in infants born in a regional perinatal unit: cause, outcome, and white blood cell response. Am J Obstet Gynecol 1987, 156(2):407-413.

28. Yoon BH, Jun JK, Park KH, Syn HC, Gomez R, Romero R: Serum C-reactive protein, white blood cell count, and amniotic fluid white blood cell count in women with preterm premature rupture of membranes. Obstet Gynecol 1996, 88(6):1034-1040.

29. Du J, Li L, Dou Y, Li P, Chen R, Liu H: Diagnostic utility of neutrophil CD64 as a marker for early-onset sepsis in preterm neonates. PLoS One 2014, 9(7):e102647.

doi:10.1186/1471-2431-14-248

Cite this article as: Chacha et al:: Utility of qualitative C- reactive protein assay and white blood cells counts in the diagnosis of neonatal septicaemia at Bugando Medical Centre, Tanzania. BMC Pediatrics 2014 14:248.

\section{Submit your next manuscript to BioMed Central and take full advantage of:}

- Convenient online submission

- Thorough peer review

- No space constraints or color figure charges

- Immediate publication on acceptance

- Inclusion in PubMed, CAS, Scopus and Google Scholar

- Research which is freely available for redistribution
C Biomed Central 